\title{
Establishing Clinical Relevance in Preclinical Alzheimer's Disease
}

\author{
R.A. Sperling, R.E. Amariglio, G.A. Marshall, D.M. Rentz
}

Center for Alzheimer Research and Treatment, Brigham and Women's Hospital and Massachusetts General Hospital, Harvard Medical School

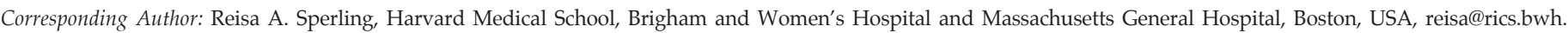
harvard.edu

Oonverging evidence suggests that the pathophysiologic processes underlying Alzheimer's disease (AD) begin more than a decade prior to dementia $(1,2)$, starting with increased amyloid deposition followed by neurodegeneration and brain atrophy (3). Moreover, consensus is emerging that our best opportunity for intervention is likely prior to widespread neurodegeneration. For an antiamyloid treatment to be effective, this may mean it will be necessary to treat the disease before symptoms appear. However, since by definition, the preclinical AD population does not have clinically detectable functional deficits, assessing the benefits of treatment becomes problematic.

Although cognition may be the earliest clinically relevant marker of disease progression, the temporal lag between the accumulation of AD pathology, cognitive decline and functional impairment remains to be elucidated. Evidence indicates that early cognitive decline is followed closely by subtle functional impairments in high-level everyday activities, suggesting that more sensitive measures of cognition and function may be useful as the best markers of early change.

\section{Assessing cognition and function in early stage disease}

Connecting the dots between current cognitive and functional tests and clinically relevant markers of disease progression may be accomplished by a combination of: 1) updating traditional measures of function, 2) including more subjective measures of cognitive function, 3) developing performance-based functional measures, and 4) making cognitive tests more clinically relevant.

Several scales have been developed to assess activities of daily living (ADL) and instrumental ADLs (IADL) in clinical trials (4). In comparison to basic ADLs such as feeding, toileting, grooming, and bathing, IADLs represent more complex activities, such as managing finances and handling medications, which tap into cognitive abilities. Basic ADLs are typically impaired in moderate-to-severe $\mathrm{AD}$, whereas decline in the ability to perform IADLs may occur at the MCI stage or earlier (5). A widely used IADL scale developed in 1997(6) has recently been updated by the Alzheimer's
Disease Cooperative Study (ADCS IADL) to include items relevant in the 21st century, such as using and remembering passwords, smartphones, and the Internet. Both study participants and partners may be assessed using IADL scales.

Subjective memory concerns have also been used to assess subtle cognitive decline in the earliest stages of the disease, and studies have demonstrated an association between amyloid burden and subjective cognitive complaints (SCC) among normal elderly $(7,8)$. A number of measures can be used to assess subjective cognitive concerns. For example, the ADCS Cognitive Function Instrument (CFI) includes both self-report and partner rating scales. The CFI is simple to administer, asking subjects and partners to compare current cognitive abilities to one year ago. For example, participants are asked "Compared to one year ago, do you have more difficulty managing money (e.g., paying bills, calculating change, completing tax forms)?" Participants may check either yes, no, or maybe; or in some instances "does not apply", and a score is calculated from these answers.

In a recent study, Amariglio et al (9) compared longitudinal scores on the CFI between "CDR progressors", i.e., subjects who progressed from CDR 0 to CDR 0.5 or 1.0, and "CDR stable", i.e., those who did not progress. CFI scores of CDR progressors separated early from the scores of CDR stable subjects and continued to increase over the four year time frame of the study, while scores of CDR stable subjects remained essentially unchanged. In the same study, the investigators showed that the CFI was able to differentiate subjects who were APOE $\varepsilon 4$ carriers from those who were not. Carriage of the ApoEc4 allele confers a substantially higher risk of developing sporadic, late-onset $\mathrm{AD}$ as well as an earlier age of onset (10). The study also examined the correlation between subjective CFI scores and objective cognitive testing, demonstrating that self and partner subjective ratings change at different rates. Initially, study partners'ratings are somewhat less correlated with the participants actual performance, but as the disease progresses and cognition worsens, CFI scores from the study partners' start to catch up. These changes may reflect the fact that individuals in the later stages of disease experience anosognosia, or lack of awareness of their impairments. The combination of self and partner 
ratings therefore appears to correlate better with the cognitive testing than either alone.

\section{Is it clinically relevant?}

Regulatory agencies require demonstration of the clinical relevance of outcome assessments. While some of the most widely used neuropsychological tests may indeed reflect neurological processes that are changing over the course of the disease, it remains a challenge to capture these early changes using clinically relevant tests. For example, connecting the dots on trail making tests may assess multiple cognitive processes such as attention, visual search, psychomotor speed, and planning (11), the relevance of this to an individual's everyday functional capacity has not been demonstrated. It may be that the best way to capture early cognitive decline with a clinically relevant scale is to employ performance-based functional assessments. Our group recently described a new performance based measure involving three highlevel, real-life automated phone menu tasks frequently encountered by seniors: calling a pharmacy to refill a prescription, calling a health insurance company to select a new primary care provider, and making a bank transfer in order to have enough money to pay taxes (12). Performance on these tasks -- both time required to complete the task and number of errors --was compared between clinically normal elderly and patients with MCI. For both of these measures, statistically significant differences were seen between the two groups. In addition, a subgroup of these subjects underwent magnetic resonance imaging (MRI), which showed that impaired performance was associated with inferior temporal cortical thinning in clinically normal subjects. This finding aligns with other studies suggesting that cortical thinning may be one of the earliest biomarker correlates of cognitive and functional decline $(13,14)$, and further supports the use of sensitive performance measures for the assessment of decline in the earliest stages of the disease.

In terms of making cognitive tests more relevant, tests of memory problems encountered in real life, such as remembering names and faces and pattern separation (ability to distinguish among very similar stimuli) may be especially sensitive in an aging population and particularly in the presence of amyloidosis (15). Moreover, for use as assessments to detect drug effects in clinical trials, repeated measures will be needed. Thus, computerized tests that provide frequent serial assessments may be especially useful. For use in the Dominantly Inherited Alzheimer's Network (DIAN) and Anti-Amyloid Treatment in Asymptomatic Alzheimer's (A4) studies, we have developed an iPad version of a computerized cognitive composite - CogState "plus" (16). This composite combines the CogState brief battery, which assesses reaction time, working memory, and incidental learning, along with face-name associative memory and behavioral pattern separation tests.

We are getting closer to closing the gap between cognitive assessments and clinical relevance, but thorny issues remain with regard to current and planned secondary prevention trials. Longer trials will be needed to demonstrate clinically meaningful change, a consequence of which will be greater attrition. Moreover, functional change using current measures appears to be non-linear, speeding up as an individual approaches the MCI stage of the disease. What that means is that these measures will be challenging to use in delayed start designs.

The real-life relevance of early functional change also needs to be more clearly demonstrated. Insurance coverage is often linked to demonstrating financial benefit rather than improving quality of life, so it will be crucial to translate early functional impairments to financial consequences, for example demonstrating how errors on filling a prescription result in increased costs from inadequate treatment.

Disclosures: Dr. Sperling has served as a consultant for Merck, Eisai, Janssen, Boehringer-Ingelheim, Isis, Lundbeck, Roche, and Genetech. Dr. Amariglio has no disclosures. Dr. Marshall has served as a consultant Halloran, GliaCure, and Janssen Research \& Development. Dr. Rentz has served as a consultant for Eli Lilly, Neurotrack and Lundbeck.

Acknowledgments: The authors are supported by the National Institute on Aging (P01AG036694; R01 AG046396; R01 AG027435; K24 AG035007; K23AG044431, K23 AG033634, and U19 AG10483), Janssen, Eisai Inc., Eli Lilly and Company, and Bristol-Myers-Squibb, Fidelity Biosciences, Alzheimer's Association (LEARN, NIRG-12-243012) and, and other philanthropic organizations. The authors wish to acknowledge the invaluable contributions of Dr. Paul Aisen, Michael Donohue, and the ADCS, and colleagues at the Harvard Aging Brain Study for contribution to this work. The authors also wish to thank Lisa Bain for assistance with the manuscript preparation.

\section{References}

1. Rowe CC, Ellis KA, Rimajova M, et al. Amyloid imaging results from the Australian Imaging, Biomarkers and Lifestyle (AIBL) study of aging. Neurobiol Aging. 2010;31(8):1275-83.

2. Bateman RJ, Xiong C, Benzinger TLS, et al. Clinical, cognitive, and biomarker changes in the Dominantly Inherited Alzheimer Network. N Engl J Med. 2012;367(9):795-804.

3. Jack CR, Jr., Knopman DS, Jagust WJ, et al. Tracking pathophysiological processes in Alzheimer's disease: an updated hypothetical model of dynamic biomarkers. Lancet Neurol. 2013;12(2):207-16.

4. Robert P, Ferris S, Gauthier S, Ihl R, Winblad B, Tennigkeit F. Review of Alzheimer's disease scales: is there a need for a new multi-domain scale for therapy evaluation in medical practice? Alzheimers Res Ther. 2010;2(4):24.

5. Marshall GA, Amariglio RE, Sperling RA, Rentz DM. Activities of daily living: where do they fit in the diagnosis of Alzheimer's disease? Neurodegener Dis Manag. 2012;2(5):483-91.

6. Galasko D, Bennett D, Sano M, et al. An inventory to assess activities of daily living for clinical trials in Alzheimer's disease. The Alzheimer's Disease Cooperative Study. Alzheimer Dis Assoc Disord. 1997;11 Suppl 2:S33-9.

7. Amariglio RE, Becker JA, Carmasin J, et al. Subjective cognitive complaints and amyloid burden in cognitively normal older individuals. Neuropsychologia. 2012;50(12):2880-6.

8. Perrotin A, Mormino EC, Madison CM, Hayenga AO, Jagust WJ. Subjective cognition and amyloid deposition imaging: a Pittsburgh Compound B positron emission tomography study in normal elderly individuals. Arch Neurol. 2012;69(2):223-9.

9. Amariglio RE, Donohue MC, Marshall GA, et al. Tracking early decline in cognitive function in older individuals at risk for Alzheimer disease dementia: The Alzheimer's Disease Cooperative Study Cognitive Function Instrument. JAMA Neurol. 2015.

10. Corder EH, Saunders AM, Strittmatter WJ, et al. Gene dose of apolipoprotein E type 4 allele and the risk of Alzheimer's disease in late onset families. Science. 1993;261(5123):921-3.

11. Salthouse TA. What cognitive abilities are involved in trail-making 
performance? Intelligence. 2011;39(4):222-32.

12. Marshall G, Dekhtyar M, Bruno J, et al. A new performance-based activities of daily living instrument for early Alzheimer's disease. AAIC. Cophenhagen, Denmark: Alzheimers Dement; 2014:P365.

13. Pereira JB, Svenningsson P, Weintraub D, et al. Initial cognitive decline is associated with cortical thinning in early Parkinson disease. Neurology. 2014;82(22):2017-25.

14. Marshall GA, Lorius N, Locascio JJ, et al. Regional cortical thinning and cerebrospinal biomarkers predict worsening daily functioning across the Alzheimer's disease spectrum. J Alzheimers Dis. 2014;41(3):719-28.
15. Rentz DM, Amariglio RE, Becker JA, et al. Face-name associative memory performance is related to amyloid burden in normal elderly. Neuropsychologia. 2011;49(9):2776-83.

16. Rentz, D, Parra Rodriguez, M, Amariglio, R, Stern, Y, Sperling, R, Ferris, S. Promising Developments in Neuropsychological Approaches for the Detection of Preclinical Alzheimer's disease: A selective review. Alzheimer's Research \& Therapy 2013; 5 (6):58. 